\title{
Evaluation of bone excision effects on a human skull model - II: finite element analysis
}

\section{Constantinos Franceskides $^{1^{*}}$, Michael Gibson ${ }^{2}$, Peter Zioupos*1}

\author{
${ }^{1}$ Musculoskeletal and Medicolegal Research Group, Cranfield Forensic Institute, \\ Cranfield University at the Defence Academy of the United Kingdom, Shrivenham, SN6 \\ 8LA, UK \\ ${ }^{2}$ Centre for Simulation \& Analytics, Cranfield University, Defence Academy of the UK; \\ Shrivenham, SN6 8LA, UK
}

\begin{abstract}
Patient-specific computational models are powerful tools which may assist in predicting the outcome of invasive surgery on the musculoskeletal system, and consequently help to improve therapeutic decision-making and post-operative care. Unfortunately, at present the use of personalized models that predict the effect of biopsies and full excisions is so specialized that tends to be restricted to prominent individuals, such as high-profile athletes. We have developed a finite element analysis (FEA) model to determine the influence of the location of an ellipsoidal excision $(14.2 \times 11.8 \mathrm{~mm})$ on the structural integrity of a human skull when exposed to impact loading, representing a free fall of an adult male from standing height. The FEA model was compared to empirical data based on the drop-tower testing of 3D-printed physical skull models where deformations were recorded by digital image correlation. In this bespoke example we found that the excision site did not have a major effect on the calculated stress and strain magnitudes unless the excision was in the temporal region, where the reduction in stiffness around the excision caused failure within the neighboring area. The FEA model allowed meaningful conclusions to be drawn for the implications of using such a technique based on what we know about such conditions indicating that the approach could be both clinically beneficial and also cost-effective for wider use.
\end{abstract}

Keywords

Biopsy; Skull Fracture; Strains; Digital Image Correlation; Impact Loading; FEA 


\section{Introduction}

The increasing capacity for computed tomography (CT) and magnetic resonance imagining (MRI) in the oncology services industry has led to a steady growth in the number of screenings per year (Table 1). In turn, the number of cancer diagnoses has increased and patient survival has improved, ${ }^{1}$ with the combined death rate in men and women falling $23 \%$ from its peak in 1991 to $2012 .^{2}$ Where biopsies or excisions are required, personalized models (in either physical or computational form) that predict the outcome of surgery are rarely employed because of the drain on public healthcare resources. Such models are usually considered only for prominent individuals such as high-profile athletes ${ }^{3}$. However, recent technological advances have raised the prospect that such personalized approaches could be based on data routinely obtained during screening, such as pre/post-operative CT scans, which could then be exploited to create computational models that predict patient-specific outcomes. One modeling approach that has been demonstrated successfully in this context is finite element analysis (FEA), which subdivides a large problem into simpler parts (finite elements) that can be solved before reassembling them into a larger model of the entire problem.

Knowledge gained from the application of FEA techniques to cellular composite structures, and specifically the mechanical properties of bones, has highlighted the utility of such a modeling approach. ${ }^{4,5}$ Although usually assumptions are made, such as simplifying the sandwich structure of bone (like in the skull, pelvis etc.) into a more homogenous material that is easier to model such tools still offer the ability to solve complex morphologies. For example, numerical simulations have been carried out to investigate three-point bending of the frontal part of the cranium, ${ }^{6,7}$ where the internal porous morphology was simulated by introducing voids in anisotropic and isotropic models. When modeling skulls, a typical approach is first to investigate their mechanical properties and then to optimize analog or other model materials to mimic the actual elastic and/or plastic response of human bone.,

In a recent experimental study we tested in impact loading the physical model of a skull of a 28-yr old male donor produced by 3D printing, by using a drop-tower testing machine and recorded its response with digital image correlation (DIC). ${ }^{10}$ The drawback of any experimental study is that it is destructive, and it can be replicated only for a small number of samples and within the ability to replicate the boundary conditions. The alternative is FEA modelling of the problem, which we attempted in a preliminary study that followed the physical tests. ${ }^{11}$ FEA models are non-destructive, they allow a test to be repeated infinitely under various conditions, and they reproduce the results for any structural problem on a global scale (strain can be read and predicted at any part of the structure) not just locally (DIC and extensometry are always relatively speaking site specific). The purpose of the present study is to explore and exploit the versatile predictive capability of an FEA modeling, an in-silico approach compared to other alternatives like hands on experimental studies using DIC or making many physical replicas with $3 \mathrm{D}$ printing. As a useful outcome of this study we set out as a task to explore the effects that a skull defect will have if is situated in various different anatomical sites on the skull. 


\section{Materials and methods \\ The patient}

The replica skulls used in this study were modelled on a 28 -year-old male patient who had no previous history of musculoskeletal disorders and no prior bone tissue surgery. The excision to the occipital skull region was performed by a consultant neurosurgeon at Wellington Hospital, London, UK, in the context of investigating the presence of a focal point of bone malignancy. The patient was otherwise healthy, but concerned that the excision would have compromised the structural strength of his skull. Ethical approval for the work was granted by the relevant Cranfield University committee and the patient consented under the approval reference CURES/787/2016.

\section{Design of the in silico skull models}

The in silico skull models were based on the same post-operative clinical CT scan acquired at a resolution of $0.488 \times 0.488 \times 1.2 \mathrm{~mm}$ and at $120 \mathrm{kV}$ and $400 \mathrm{~mA}$ (Figure 1, step 1). ${ }^{10}$ Five digital skull geometries were created: the "original" model with a lower occipital excision, a "healthy" control with the excision filled in, and three variants with the excision moved to the parietal, frontal or temporal region (Figure 1, step 2). All scans were imported to Simpleware ScanIP v7 as a 32-bit float, but were converted to an 8-bit float and resampled (using the cubic function) to match the voxel axial dimension to the in-plane dimension with the resulting cubic voxels of side length $0.488 \mathrm{~mm}$. A series of masks was then applied, with the appropriate grayscale thresholding and flood fill operations to select only bone matter within the scanned volume. The resulting model was passed through a Gaussian smoothing filter to better represent the actual skull geometry (voxel-based output from CT scanners is dependent on the slicing interval, thus creating a stepped appearance). Further cavity-fill and morphological close operations were conducted before exporting the model stl file.

The "healthy" skull geometry was created using the neighboring morphology, such as the surrounding bone thickness and curvature, thus mimicking as closely as possible the preoperative condition of the region. The "original" skull model with the lower occipital excision was used to create the remaining geometries with alternative excision sites by first creating an intermediate skull with the excision patched, and then duplicating the geometry of the excision by subtracting the initial skull geometry from the initial patch geometry. This excision segment could then be moved to any point on the intermediate skull to create a new excision site. The tapering of the excision remained constant with respect to the excision diameter of $14.2 \pm 0.5 \mathrm{~mm}$, thus eliminating thickness variations at the different excision sites.

\section{Preparation of the physical skull models ${ }^{10}$}

Six skulls were printed in acrylonitrile butadiene styrene (ABS) using a fused deposition modeling (FDM) Stratasys Uprint 3D printer (Figure 1, step 3). The .stl files were imported into the CatalystEX proprietary software, which was used to create the .cmb tool-path files to control the printing process. Support fill was set to 'smart', the model interior to 'solid' and the layer resolution to $0.254 \mathrm{~mm}$. The printing of each model was 
completed in $\sim 42 \mathrm{~h}$ and required almost a full reel of ABSPlus-P430 material (33 cubic inches). The freshly printed skulls were placed in a Support Cleaning Apparatus 1200 and washed with a cleaning solution containing sodium hydroxide (WaterWorks) at $70^{\circ} \mathrm{C}$ for $2 \mathrm{~h}$ to dissolve any remaining support material. Perma-Gel was used to fill the models in order to mimic the properties of the brain and contribute by its presence towards a more reliable energy absorption and response for the skull as a whole. Perma-Gel was chosen because of its characteristics seen in dynamic tests in the past. ${ }^{12}$ A random speckle pattern was then applied to make the surface of the skull univocally identifiable. ${ }^{13-15}$ The black-to-white ratio was kept as close as possible to 1:1, and Castrol White Contrast Paint 710 (2386) non-strippable aerosol was used to apply the high-contrast background evenly over the model surface. Finally, the size of the speckles was determined using the following equations ${ }^{16,17}$;

$$
\begin{gathered}
S_{\text {ideal }}=\frac{4 \pm 1}{M} \\
M_{\text {factor }}=\frac{n_{\text {length sensor pixels }}}{d_{\text {length }}}
\end{gathered}
$$

The ideal speckle pattern size is expressed as 3-5 pixels divided by the magnification factor $M$, i.e. the ratio of the number of pixels along the length of the sensor to its physical length.

\section{Drop tests}

All six skull models were subjected to a load on the occipital region mimicking the effect of an unconscious or incapacitated human falling backwards and striking this region on a hard surface. ${ }^{10}$ An Imatek IM10 drop tower was used to deliver the impact with a total carriage and striker mass of $2.91 \mathrm{~kg}$ (Figure 1, step 4) ${ }^{10}$. The $45-\mathrm{mm}$ circular titanium striker was fitted with a dampening surface adapted from a Humanetics Hybrid III headform thickness of $12.7 \mathrm{~mm}$ with $43 \pm 5$ shore hardness ' $\mathrm{A}$ ' to provide a dampening effect (smoother ramp rate) similar to the soft tissues surrounding a real human skull. Images were captured using two V12.1 Phantom high-speed cameras, recording at 15,000 fps, and PCC standalone software.

The data were analyzed by DIC using Aramis GOM software with quadrangle facets at 19 pixels (size) and 17 pixels (step), 484 3D points generated over 176 stages, and an average intersection deviation of $0.0150 \mathrm{~mm}$. Artificial lighting was provided by three light sources: two conventional halogen lights with focal adjustment, and a light emitting diode (LED) Cree floodlight acting as a background source. Due to space limitations, the LED source was reflected onto the object using a flat mirror placed outside the impact area. Two series of tests were conducted, with varying striker velocities of $\sim 8 \mathrm{~m} / \mathrm{s}$ at 100 $\mathrm{J}$ and $\sim 9.75 \mathrm{~m} / \mathrm{s}$ at $130 \mathrm{~J}$. These speeds are for the striker not the speeds by which the skull would impact the ground in free fall. The striker speeds were chosen to match biomimetically the energies in a free fall case. ${ }^{10}$ The lower-energy impacts were used to determine the loading response under the defined testing analogy $\left(50^{\text {th }}\right.$ percentile male adult losing consciousness and falling with direct impact on the occipital region), and the higher-energy impacts were used to induce failure in the skulls and thus determine the 
fracture pattern and propagation path. We must note that the present set up involves a striker hitting a skull which was securely held on a semi-soft substrate. In real world scenarios it is rather a free-falling skull hitting for instance a hard surface. However, using in-lab equipment and the instrumented drop tower to drive a striker towards a skull allows a degree of control over a number of other confounding test variables. Being able to record and impact the same area was more important and prominent in understanding the load dissipation proximal to the excision.

\section{Finite element modelling}

The five skull geometries described earlier were used to create the volumetric meshes required for FEA. These differed from the previously generated surface meshes (.stl files) in that the volume of each skull was divided into a relatively large number of small, geometrically simple, polyhedral elements (typically tetrahedra and hexahedra). ScanIP has two principal methods for generating meshes, FE-Free and FE-Grid. The former creates a free-form mesh of defined geometry, whereas the latter maps cubic (perfect hexahedral) elements directly onto the voxels defined by the scan, typically generating a much higher element count. The in silico models generated for the purposes of this investigation were meshed using the FE-Free method, with tetrahedral elements and setting the mesh refinement to -20 . The meshes were then exported individually as .cdb files. An example of such a mesh is shown in Figure 2, rotated to show the excision site.

\section{Simulation}

The static, structural simulation of the drop-tower test was conducted using ANSYS Mechanical APDL v17.1 FEA software. The FEA models were created by importing the meshes (see above) and applying the appropriate material properties, loads, constraints and solution settings to best match the experimental conditions, as previously reported for similar loading scenarios, impactor velocities and impact energies. ${ }^{18}$ During the modeling phase, the loading was considered to be quasi-static within the FEA model and was applied uniformly over the striker impact area on the rear of the skull, offset to the occipital lobe, with the load set as equal to the peak striker force measured experimentally. Two main constraint areas were selected: (1) the surface of the face, primarily to restrain forward motion, and (2) the lower surface of the skull. Each of the five skull variants was constrained and loaded in the same manner such that only the mesh varied. A constant impact force was applied, equal to the peak recorded impact force of the empirical tests, i.e. $\sim 10,000 \mathrm{~N}$ as previously reported. ${ }^{18,19}$ To estimate the associated duration of impact, the change in momentum of the striker was equated to the impulse applied to the skull, as shown in the following equation with $F$ representing the force, $m$ the mass, $\Delta v$ the velocity change, $I$ the impulse and $t$ the time:

$$
I=m \Delta v=F t
$$

Therefore, assuming a constant deceleration force, the duration of deceleration (from a recorded speed of $4.6 \mathrm{~m} / \mathrm{s}$ ) may be estimated as follows:

$$
t=m \Delta v / F
$$

which resolves to: 


$$
t=\frac{2.91 \times 4.6}{10000}=0.00134 \mathrm{~s}
$$

The high-speed video used during the experimental portion of this investigation was recorded at 15,000 fps. Impact lasted 25 frames $(1.67 \mathrm{~ms})$ indicating that the force applied was consistent with the represented change in momentum.

The ABS material was not expected to shatter when struck, but it was expected to undergo plastic deformation with little strain hardening. Accordingly, a bilinear, kinematic material model was used to represent it. The material properties required by the model, and the corresponding values used in this investigation, are listed in Table 2. The front face of the skulls was constrained in a distributed, flexible manner, to represent the PermaGel restraint block in the mechanical tests. The flexible constraint was achieved by applying a large number of spring elements $(14,800)$ over the constrained surface, the first nodes of which were shared with a node on the face of the skull mesh, whereas the other end was fully constrained. Each spring element had a stiffness of $0.62 \mathrm{~N} / \mathrm{mm}$ and an initial length of $5 \mathrm{~mm}$. The lower surface of the skulls was constrained vertically, preventing rotation about the anterior-posterior and medial-lateral axes. The skull was therefore restrained during impact without causing localized stress concentrations, allowing the applied impact load to replicate a moving skull striking an obstacle. Each analysis was broken down into 10 load steps, each initially configured to solve with three sub-steps and with upper and lower limits of 15 and 2 sub-steps, respectively.

\section{Comparison of experimental and FEA model data}

The response of the FEA model was assessed against that of the ABS analog by comparing three metrics: (1) impact site displacement; (2) maximum and minimum von Mises strains proximal to the excision site; and (3) the crack initiation site. Additional trends among the FEA models of the different excision sites were assessed by comparing force, stress and strain data on a local and global basis.

\section{Summarized test methodology}

Patient-specific computational models work along the steps depicted in the methodology flow diagram of Figure 1. They involve scanning of a patient, importing the data on a PC for FEA analysis, mechanical testing of a physically created 3D printed model to record real world strains, comparison to the virtual FEA strains and in circular process which involve model refinement in its various subcomponents for optimization and validation. The present work shows the added value of the FEA approach by examining the potential effects of bone biopsies when these are placed in various sites on the skull. In reality a skull (and a physical model of one) will have a biopsy in a certain area and it can demonstrate effects for this surgical intervention in this one site alone. The one site effect has been looked in a previous experimental study, ${ }^{10}$ which used DIC, mechanical testing and 3D printing. The present work adds an implementation of FEA and after validation of the fracture pattern in the configuration that has been physically tested ${ }^{10}$ looks at the would-be effect of potential excision biopsies at other different sites on the skull.

Results and discussion

FEA mesh sensitivity 
Global and near-excision peak stress data for the occipital excision are plotted in Figure 3. Overall, the stress patterns and global peak stresses were very similar between meshes, whereas the near-excision stresses showed more mesh sensitivity. Accordingly, elementsize sensitivity analysis was conducted based on the peak near-excision stresses. This revealed that the $\sim 500,000$ element mesh generated sufficiently similar results to the $\sim 900,000$ element mesh, and the model was therefore gauged to be mesh-insensitive beyond that point. The $\sim 500,000$ element mesh corresponded to a refinement value of -20 within the ScanIP FE-Free meshing algorithm. In this study only tetrahedral elements were used and the in-built routines in ScanIP which practically allow mesh quality implementation by fine tuning of density, aspect ratios, maximum corner angles and size of elements in stress concentrating regions and so conserving element number in smooth regions.

\section{Comparison of impact site displacements}

The displacement of the two sets of skulls (excised and non-excised) in response to impact loading is shown for the physical model (DIC data) and the FEA model in Figure 4. The FEA data were extracted as the mean displacement of the impact site in response to the applied impact load, whereas the DIC data were extracted from incidence displacements (of the skull) in the associated frame sequence of the impact site. The FEA model showed a smooth displacement curve, as it is a solid model with idealized contact and continuity between the subcomponents with a gradual transition from 0 load to $10 \mathrm{kN}$. The DIC displacement curves showed a noticeable non-linear response where the two elastomer interfaces (the PermaGel substrate and the skin simulant on the impactor) both yielded at low loads ('S' type load/displacement curve typical of elastomers) before compacting and transferring the load into the skull between them. Therefore, the traces for FEA and DIC are not truly comparable because the FEA model did not include two elements on the two boundaries that can simulate the accurately the behavior of the elastomer. Once the elastomers hardened the two traces converged to the same point for force $(10 \mathrm{kn})$ and displacement $(7 \mathrm{~mm})$ and it is at this point where the simulation and experiment results were actually compared and contrasted to each other. This model aims to show qualitatively that the final fracture behaviour can be reliably inferred from deformation concentrations observed in the simulation for the elastic response region. Validation was in the form of pattern of strains, simulated and observed through DIC in this fast impact loading scenario. Appreciation of the strains was through DIC and not by microstrains on the physical model surface (as it would normally be). The DIC offers great advantages and it is the way of the future for awkward shaped objects, fast moving events and difficult tests in the field. Consequently, the validation of Figure 4 cannot be a one-to-one this would be for a static model with microstrains vs FEA values of the same. In the structural model with the boundary conditions and the substrates used the coincidence of the Force/displacement at a certain force magnitude of our interest $(10 \mathrm{kN})$ is the required outcome.

\section{Comparison of excision-proximal strain}


In terms of von Mises strain comparisons (Figure 5) there was a noticeable variation between the DIC and FEA techniques. This was anticipated, because the FEA model does not account for moments of inertia effects or thermal softening. In addition, FEA data are based on volume analysis whereas DIC can only record surface strain. As for the actual strain behavior, the different behavioral trends between the two skulls were evident, with an almost 20-fold difference between the maxima and minima. These data indicate that an excised skull experiences a greater range of strains (because of the magnitude of the strains around the excision) than the healthy skull. This showed that the structural effects may have a more profound effect on the strain patterns which add to any underlying material property effects (compromised bone tissue modulus itself). A recent application $^{20}$ which combined computational/experimental approach on the outer cortical layer of adult human calvariums has shown that while the effective failure stress and strain are related to skull position, the bone modulus itself relates to the calvarium bone quality. Results for an approach like ours, therefore, should translate well for 'nominal healthy' individuals for whom they have been produced, any material level alterations will inevitably further compound these effects.

\section{Comparison of crack initiation sites}

On the lower edge of the occipital excision site, the localized von Mises stress was $\sim 15.7$ MPa compared to only $11.8 \mathrm{MPa}$ in the same region of the healthy skull. This result indicated that the excision increases the regional stress but does not affect the overall strength of the skull. The results from the 500,000 element FEA mesh were compared with empirical equivalents, revealing that the areas of plasticity within the FEA model correlated accurately with the regions that had cracked within the printed skull, including crack initiation at the lower edge of the occipital region on the impact site (Figure 6). It must be noted that the present article is a continuation of a previous empirical study

where the cracks were observed at the impactor site ${ }^{10}$. The cracks were seen to converge to the impact site and were developing in the same pattern in both the intact (no hole) and the compromised (with excision hole) skulls. Here it is shown that initiation of the precursor crack was at the base of the skull and this can be predicted by FEA and demonstrated in the ABS physical model too. The FEA simulation, of course, predicts the behavior of the printed ABS skull within the elastic region and revealed moderate degrees of plasticity, but once a crack initiated the simulation became less accurate and stress continued to increase with strain, albeit at a low modulus. The printed ABS medium also included a degree of laminar behavior which was not replicated in the FEA model.

\section{Comparison of stress and strain}

The maximum excision-proximal stress at the frontal, parietal and temporal excision sites was consistently similar in magnitude, whereas less stress was experienced at the occipital excision site and the least stress was experienced by the intact, healthy skull (Figure 7). However, the maximum excision-proximal strain was highest by far for the temporal excision, with the other skulls showing much lower values (Figure 8). This may reflect the relatively thin bone surrounding the temporal excision, whereas the other 
excisions feature much thicker peripheral bone. The profiles of global mean strain (Figure 9) and global mean stress (Figure 10) were similar to the maximum strain, with the temporal excision showing the highest values and the other four models showing lower values but with the same ranking: parietal, frontal, occipital, normal. The unique behavior of the temporal excision may again reflect the relative thinness of the skull in that region, in sharp contrast to the lowest levels of regional and global stress and strain observed in the normal, healthy skull.

\section{Conclusions}

In the present article we have examined the behavior of an in silico skull model under load testing, with excision in different places but at a common impact site. We have examined the constraint methodology (providing firm support for the skull specimen) and then determined the ability of FEA to predict the behavior of the 3D-printed models. We used DIC to study ${ }^{10}$ phenomenologically the strain distribution (and by implication the stress concentration) in the presence and absence of the excision hole, and compared this to FEA results obtained by modelling. Our comparison of DIC and FEA testing methods indicated strong qualitative (in behavior, mapping of strains and final result pointing to a crack site) agreement between the two approaches, but there retains a lack of refinement because the DIC is good for mapping whole objects but it is not ideal for validating an FEA model the way we know it in classical mechanics. A number of micro strain gauges for instance would have been able to provide site for site comparison between observed and modelled strains for true model validation. However, the present application can enlighten us on matters such as stress concentration and crack initiation sites, which were easily identified by FEA and DIC mapping. In the testing of the physical skull ${ }^{10}$ the surgical excision hole did appear to influenced the propagation and distribution of stress and strain as well as the mechanical response of the skull. The excision hole was seen to increase the strain in the surrounding region, but it was not enough to induce a structural failure originating from the hole. This was confirmed in the present tests now involving FEA method. The present in-silico models have indicated that location of the excision in relation to the site of impact can have a significant effect on strain magnitudes. Although our data are patient specific, the methodology can be used as a basis for further research into the effect of invasive surgeries and their accompanying computer models, not only on the skull but also on the rest of the musculoskeletal system.

\section{Acknowledgments}

We acknowledge the work, skill and expertise offered by Karl Norris in the mechanical workshop of Cranfield University; Jolyon Cleaves of Vision Research for providing the high-speed cameras. Ethical approval was granted by Cranfield University Research and Ethics committee (CURES). This paper is dedicated to one of the authors, Dr Mike Gibson, whose untimely death is a great loss to us all.

\section{Funding and conflicts of interest}

This work was supported by an internal grant of Cranfield University (CF) and project grant from RCDM Birmingham (PZ) and by in-kind support from Vision Research. The authors have no conflict of interest to declare. 


\section{Data accessibility}

The data in this manuscript is available through the Cranfield University CORD data depository and preservation system at https://cranfield.figshare.com.

\section{Authors' agreement and declarations}

All authors contributed to this research and to the preparation of the manuscript.

\section{Authors' contributions}

Conceptualization: CF; Data curation: CF, MG; Formal analysis: CF, MG; Funding acquisition: CF, PZ; Investigation: CF, MG; Methodology: CF, MG, PZ; Project administration: CF, MG, PZ; Resources: CF, MG; Software: CF, MG; Supervision: MG, PZ; Validation: CF, MG; Visualization: CF; Writing (original draft): CF; Writing (review and editing): $\mathrm{CF}, \mathrm{MG}, \mathrm{PZ}$.

\section{References}

1. Office for National Statistics. Cancer survival in England: Patients diagnosed between 2010 and 2014 and followed up to 2015. Newport, UK: ONS, 2016.

2. National Cancer Institute. Statistics at a glance. https://www.cancer.gov/aboutcancer/understanding/statistics (2018, accessed 2 May 2018).

3. Hood, S., McBain, T., Portas, M., Spears, I. Measurement in Sports Biomechanics. Measurement and Control, 2012; 45(6), 182-186. doi.org/10.1177/002029401204500604

4. Verhulp E, et al. Micro-finite element simulation of trabecular-bone post-yield behaviour effects of material model, element size and type. Comp Meth Biomech Biomed Eng 2008; 11:389-395.

5. Bevill $\mathrm{G}$ and Keaveny $\mathrm{T}$. Trabecular bone strength predictions using finite element analysis of micro-scale images at limited spatial resolution. Bone 2009; 44:579-584.

6. Rahmoun J, et al. Characterization and micromechanical modeling of the human cranial bone elastic properties. Mech Res Commun 2014; 60:7-14.

7. Boruah $\mathrm{S}$ et al. Response of human skull bone to dynamic compressive loading. Gothenburg, Sweden: IRCOBI, 2013.

8. Falland-Cheung L et al. Investigation of the elastic modulus, tensile and flexural strength of five skull simulant materials for impact testing of a forensic skin/skull/brain model. $J$ Mech Behav Biomed Mater 2017; 68:303-307.

9. Garcia-Gonzalez D et al. On the mechanical behaviour of PEEK and HA cranial implants under impact loading. J Mech Behav Biomed Mater 2017; 69:342-354.

10. Franceskides $\mathrm{C}$ et al. Evaluation of bone excision effects on a human skull model $-\mathrm{I}$ : mechanical testing in impact and use of digital image correlation. Proc Inst Mech Eng, Part H: J Eng Med 2018; (In Press)

11. Gibson M, Franceskides C and Zioupos P. Simulated impact response of a 3D printed skull, with an ellipsoidal excision, using finite element analysis. 22nd Congress of the ESB, July 10-13, Lyon, France.

12. Appleby-Thomas GJ, Fitzmaurice B, Hameed A, et al. On differences in the equation-ofstate for a selection of seven representative mammalian tissue analogue materials, Journal of the Mechanical Behavior of Biomedical Materials, 2018; 77 586-593.

13. Vassolera JM and Fancelloa EA. Error analysis of the digital image correlation method. Asoc Argentina de Mecán Comp 2012; 29:6149-6161. 
14. Pan B, Lu Z and Xie H. Mean intensity gradient: An effective global parameter for quality assessment of the speckle patterns used in digital image correlation. Optics Lasers Eng 2010; 48:469-477..

15. Lecompte $\mathrm{D}$ et al. Quality assessment of speckle patterns for digital image correlation. Optics Lasers Eng 2006; 44:1132-1145.

16. Palanca M, Tozzi G and Cristofolini L. The use of digital image correlation in the biomechanical area: a review. Int Biomech 2015; 3:1-21.

17. Sutton MA, Orteu JJ and Schreier H. Image correlation for shape, motion and deformation measurements. New York: Springer, 2009

18. Asgharpour $\mathrm{Z}$ et al. The validation and application of a finite element human head model for frontal skull fracture analysis. J Mech Behav Biomed Mater 2014; 33:16-23.

19. Pinnoji PK and Mahajan P. Finite element modelling of helmeted head impact under frontal loading. Sādhanā 2007; 32:445-458.

20. Boruah $\mathrm{S}$ et al. Influence of bone microstructure on the mechanical properties of skull cortical bone - A combined experimental and computational approach. $J$ Mech Behav Biomed Mater 2017; 65:688-704. 
Table 1 - Annual growth in screening procedures. CT = computed tomography; $\mathrm{MRI}=$ magnetic resonance imaging; Pet-CT = positron emission tomography-computed tomography.

\begin{tabular}{|l|l|l|l|l|}
\hline Year & Total growth \% & CT & MRI & Pet-CT \\
\hline $2013 / 14$ & 5.1 & 13.0 & 11.3 & 5.9 \\
$2014 / 15$ & 4.9 & 11.1 & 10.5 & 18.5 \\
$2015 / 16$ & 2.1 & 6.2 & 6.7 & 9.9 \\
$2016 / 17$ & 0.9 & 5.1 & 6.1 & 32.5 \\
\hline Average & 3.3 & 8.9 & 8.7 & 16.7 \\
\hline
\end{tabular}

Table 2 - Material specifications of acrylonitrile butadiene styrene (ABS). Values for Young's modulus and yield stress were supplied by Stratasys Ltd. For comparison bone properties at the tissue/micromechanical bone material level, not those of the sandwich like structure of the skull where porosity and architecture reduce the stiffness at the structural level to one similar to that of a solid ABS layer.

\begin{tabular}{|l|l|l|}
\hline Property & ABS & Bone \\
\hline Young's modulus (E) & $2200 \mathrm{MPa}$ & $18000 \mathrm{MPa}$ \\
Poisson's ratio & 0.30 & $0.30-0.35$ \\
Yield stress & $31 \mathrm{MPa}$ & $90 \mathrm{MPa}$ \\
Plastic modulus & E/1000 $=2.2 \mathrm{MPa}$ & E/20 1 MPa \\
\hline
\end{tabular}




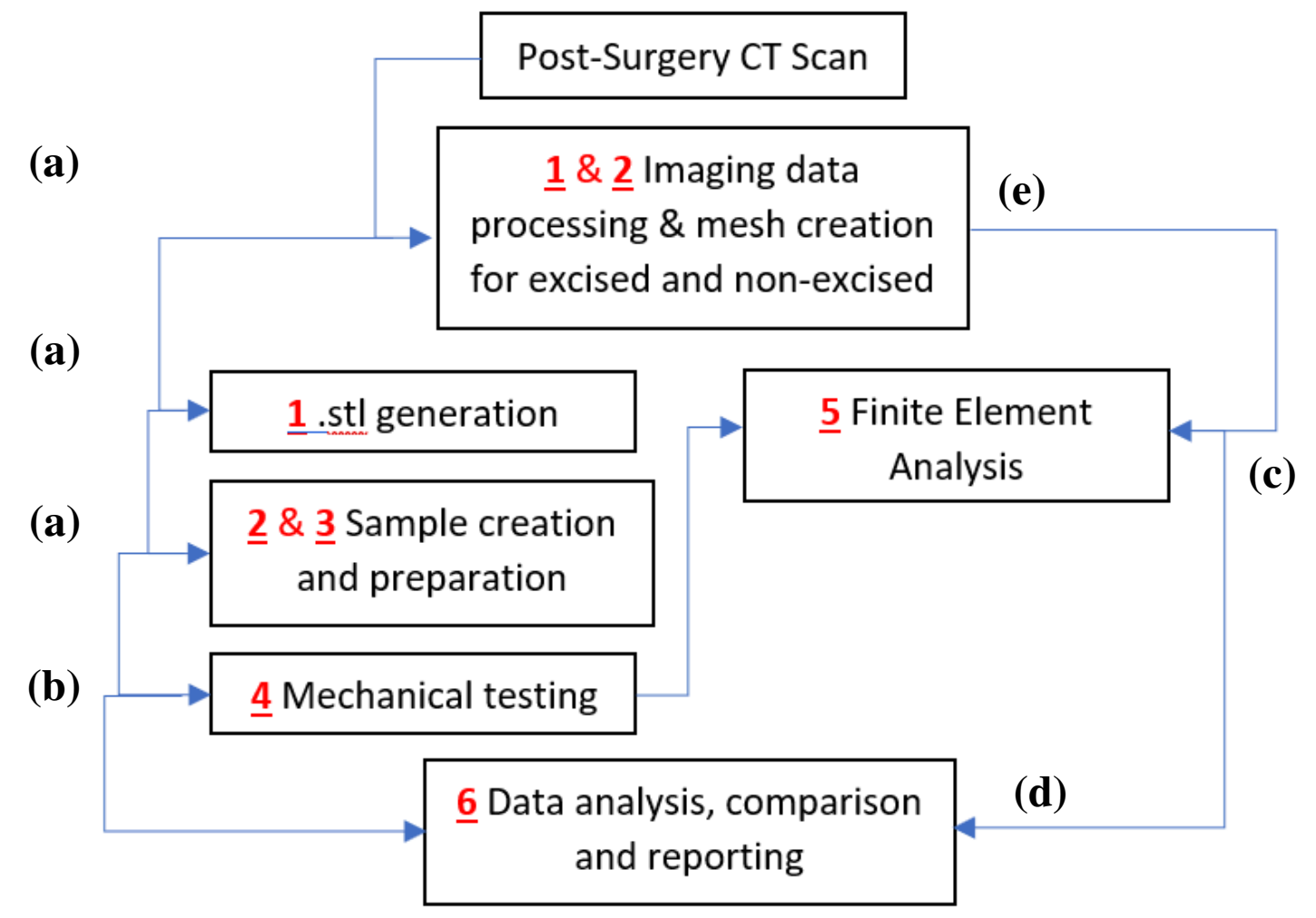

Figure 1. Combined methodology flow diagram. 1. Creation of 3D geometry of excised skull from the patient's CT scan data; 2. Duplicate 3D model and fill the excision site to approximate initial, non-excised state; 3. Creation of 3D models and accompanying meshes for various excision sites; 4 . For each of the two 3D geometries: (a)Create 3Dprinted replicas of the excised and non-excised skulls from ABS. (b)Mechanically test the 3D-printed replicas from point 2. (c)Simulate impact of the drop hammer, considering the peak load as quasi-static. (d)Compare FEA to mechanical testing. (e)Re-run FEA to investigate the effect of different excision sites. 

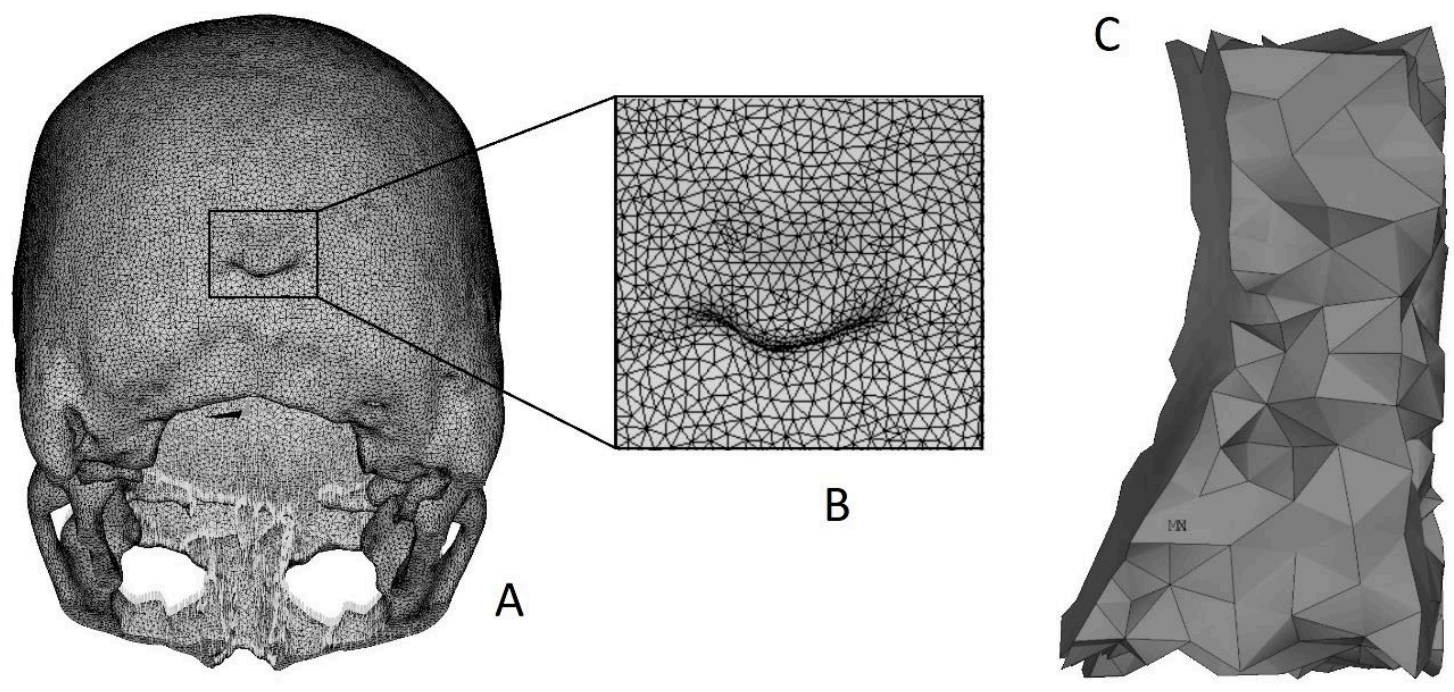

Figure 2. A. Sample skull mesh used for the finite element model. B. Enlarged area to show the tetrahedral elements; C. Cross section of the model through the skull thickness. 


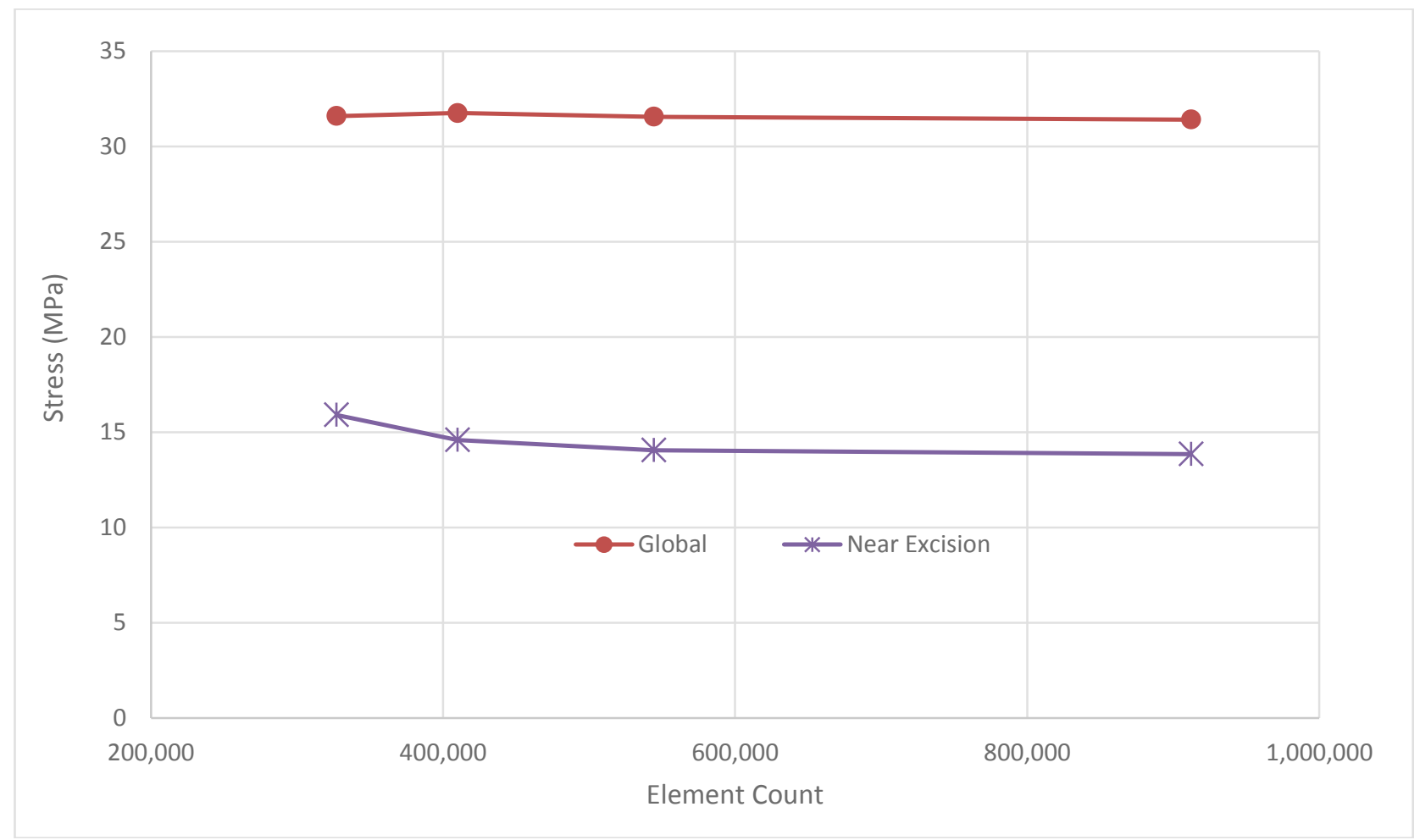

Figure 3. Global and near excision peak stresses within the skull FEA model, identifying sensitivity of elemental count. 


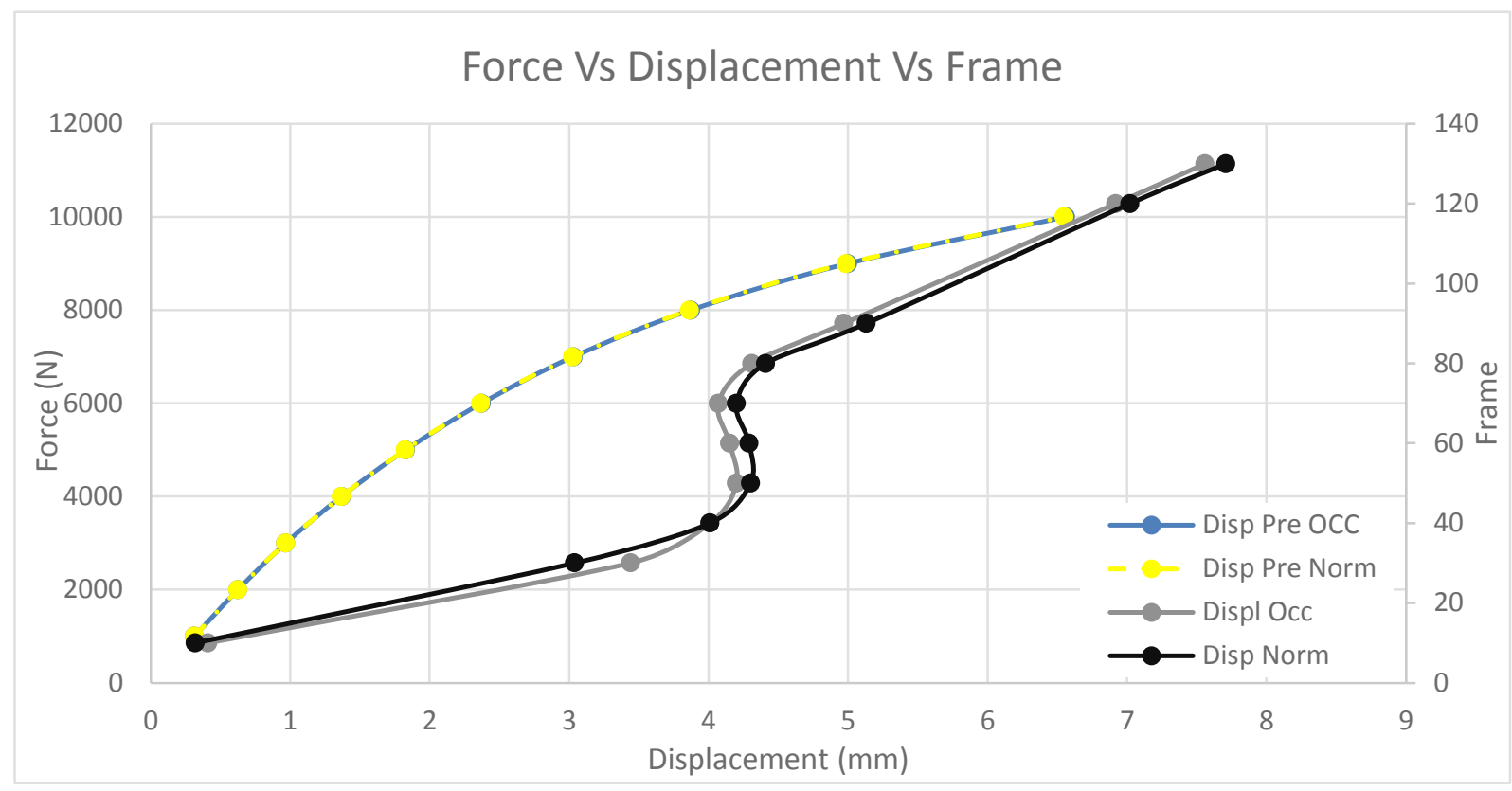

Figure 4. Overall (end to end) displacements for the FEA (dashed lines) and DIC (solid lines) skull models for two cases: with a hole at the occipital region (OCC) and an intact skull/no hole (Norm). The solid FEA model shows an incremental response while the actual physical model which contains elastomer layers on either contact surfaces (through which the forces are applied on the skull) shows a ' $\mathrm{S}$ ' like elastomeric curve. Once the elastomers have been compressed and hardened both models converge for a max impact load of $10 \mathrm{kN}$ as envisaged for the free fall situation. 


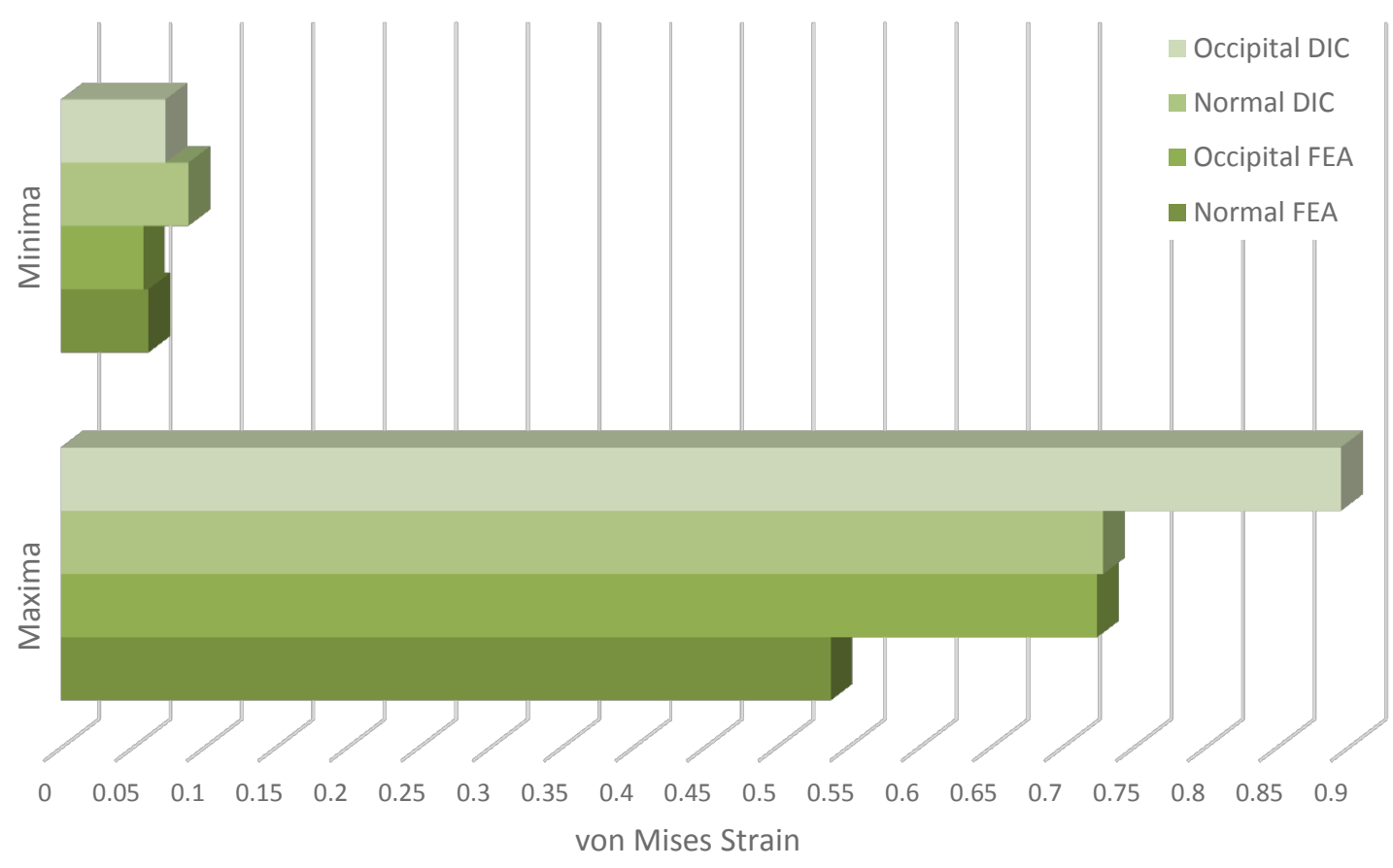

Figure 5. Comparison of proximal von Mises strain in excised and normal skulls analyzed by DIC and FEA. 

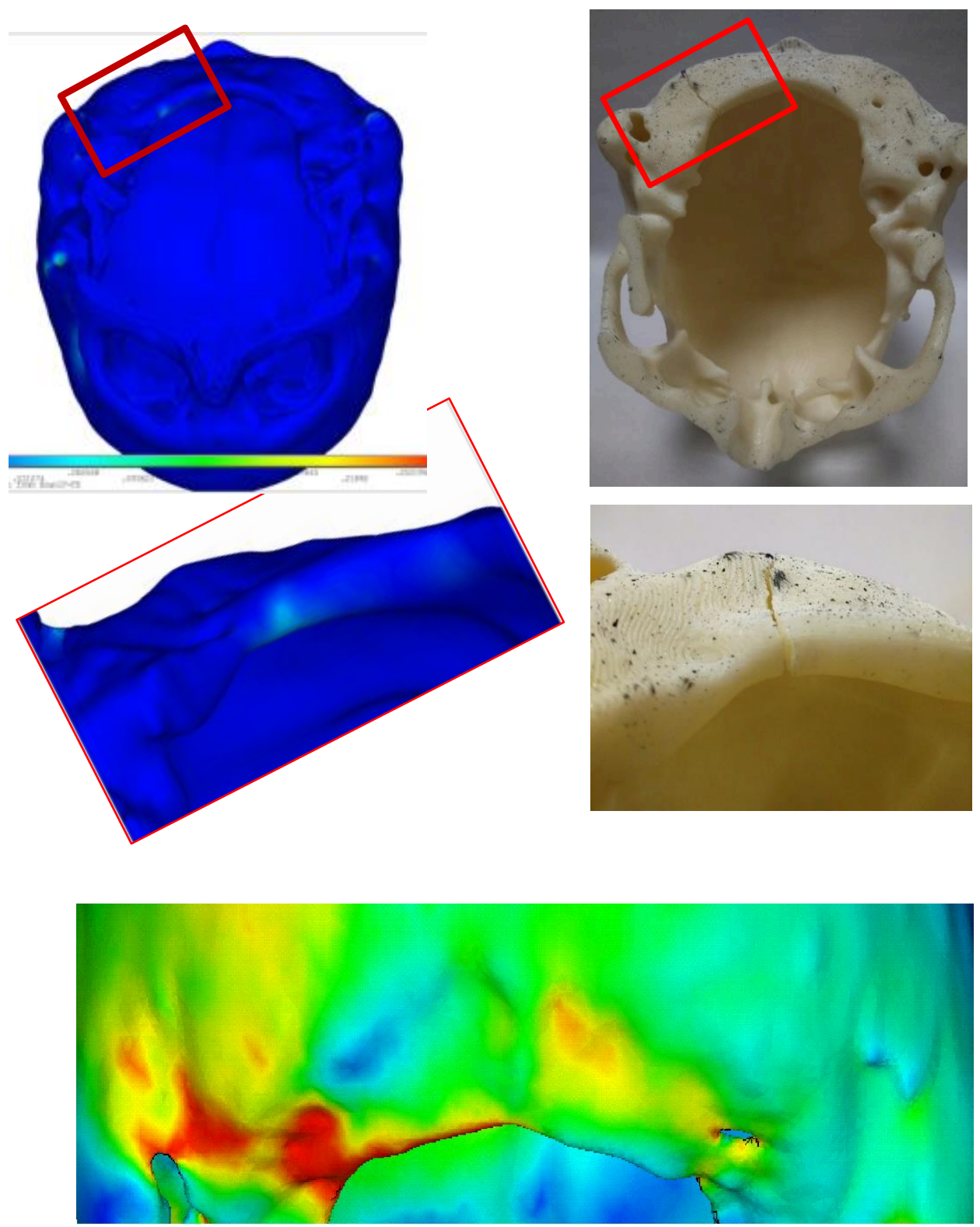

Figure 6. Maximum plastic strains in the simulated skull compared to failure site of the 3D-printed skull. Small insert for strains at an early loading stage (light blue) which progressively increased in magnitude (yellow/orange/red) as the severity of the impact loading increased. 


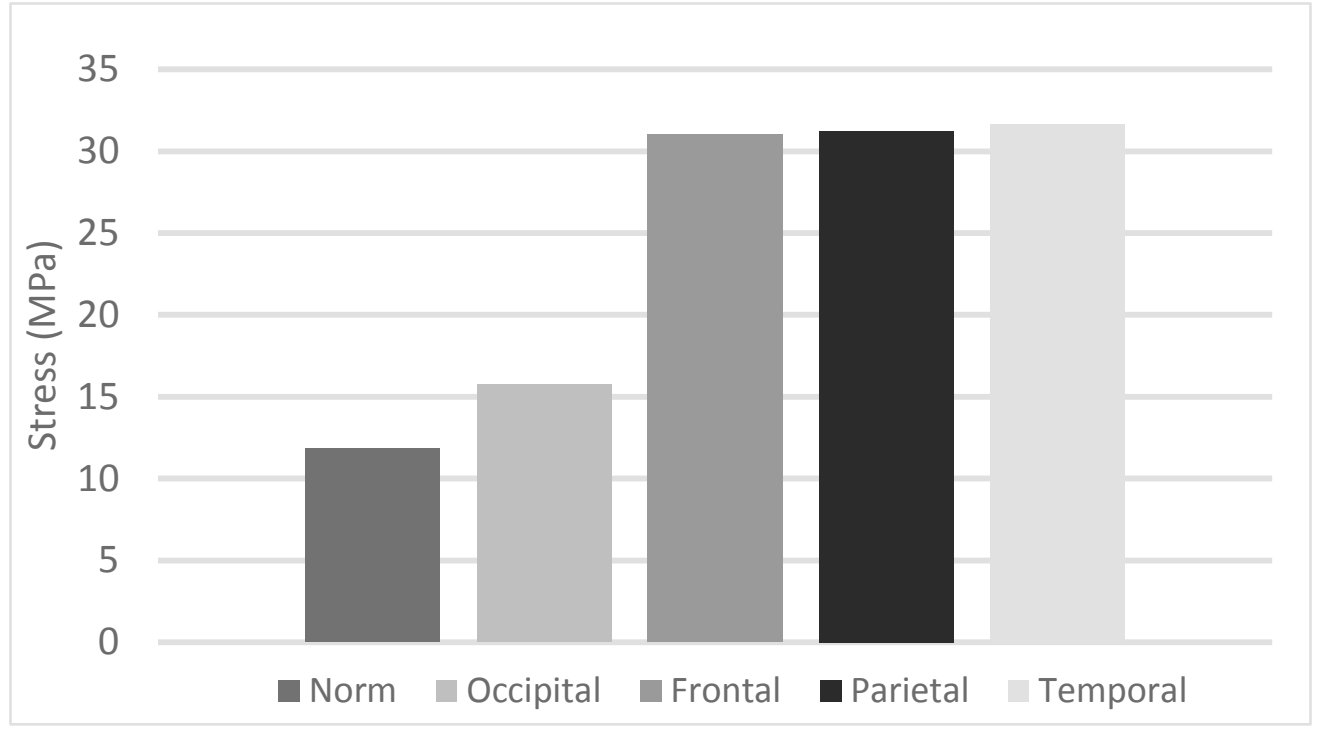

Figure 7. Maximum excision-proximal von Mises stress of the different testing sites. 


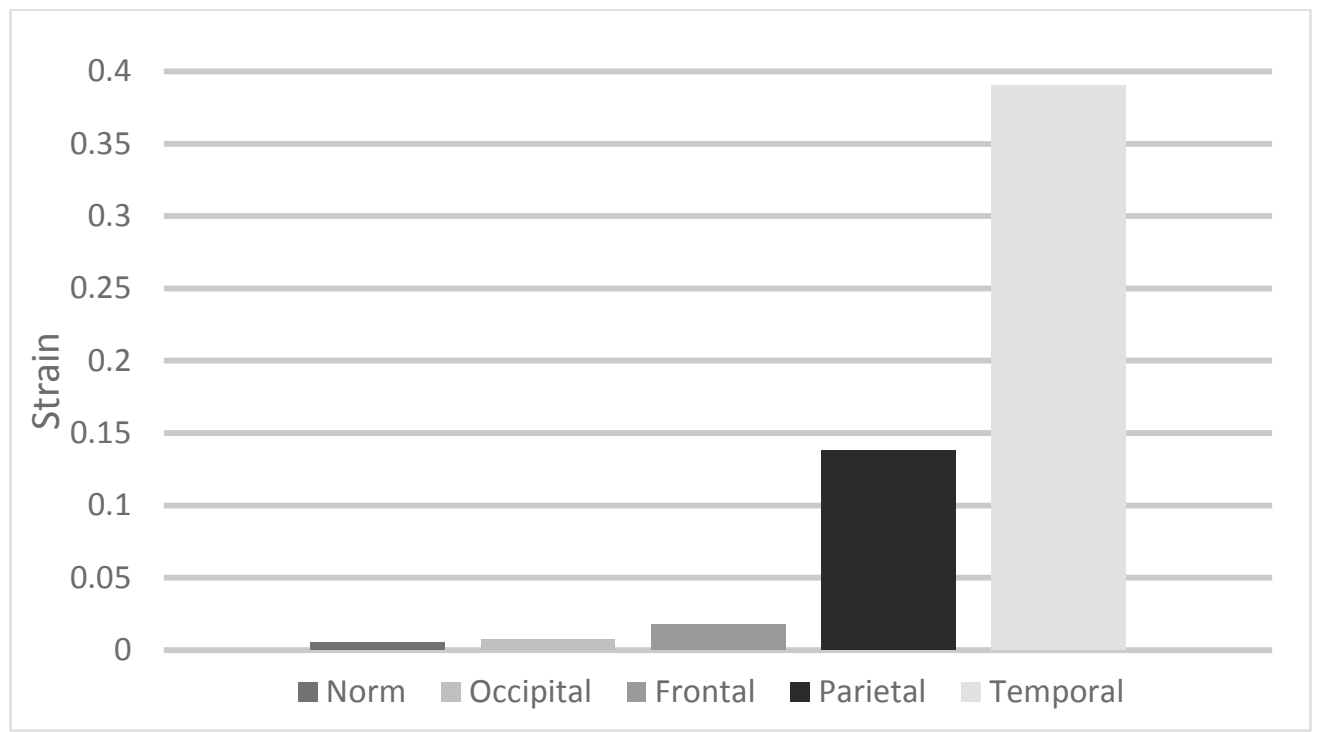

Figure 8. Maximum excision-proximal von Mises strain of different testing sites. 


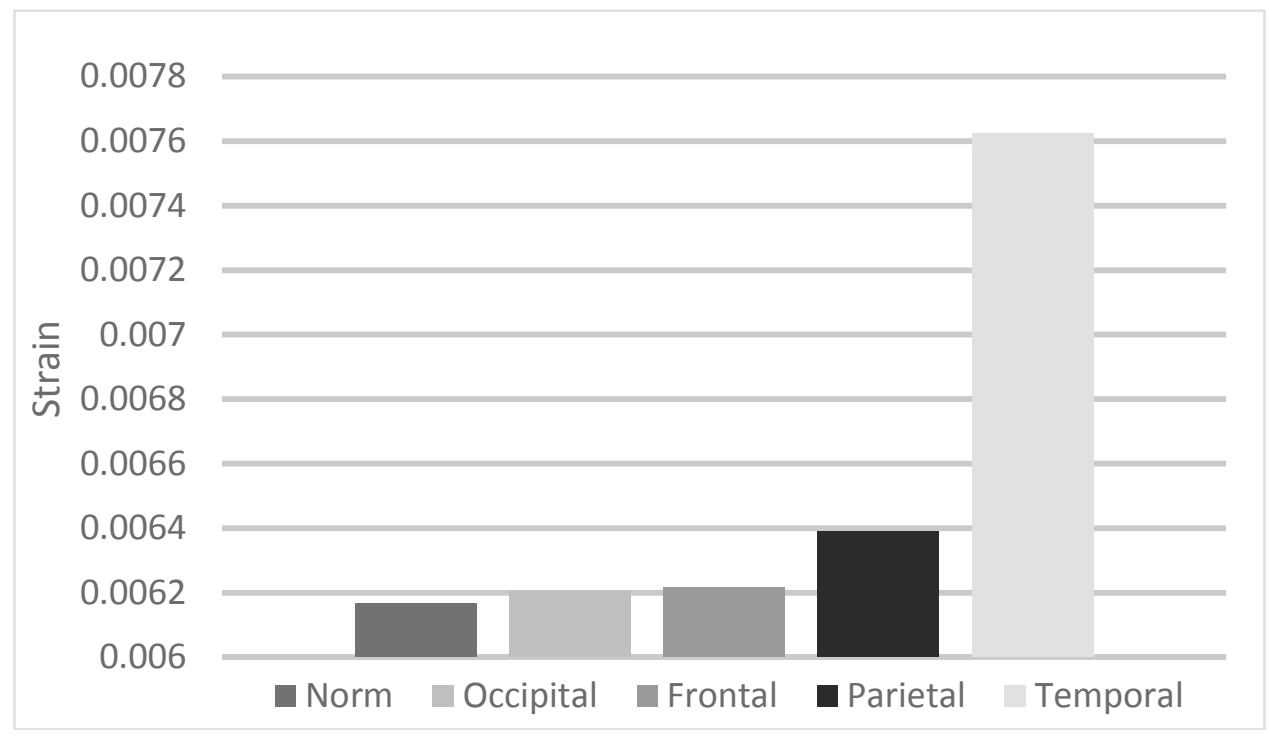

Figure 9. Global Mean von Mises Strain of different testing sites. 


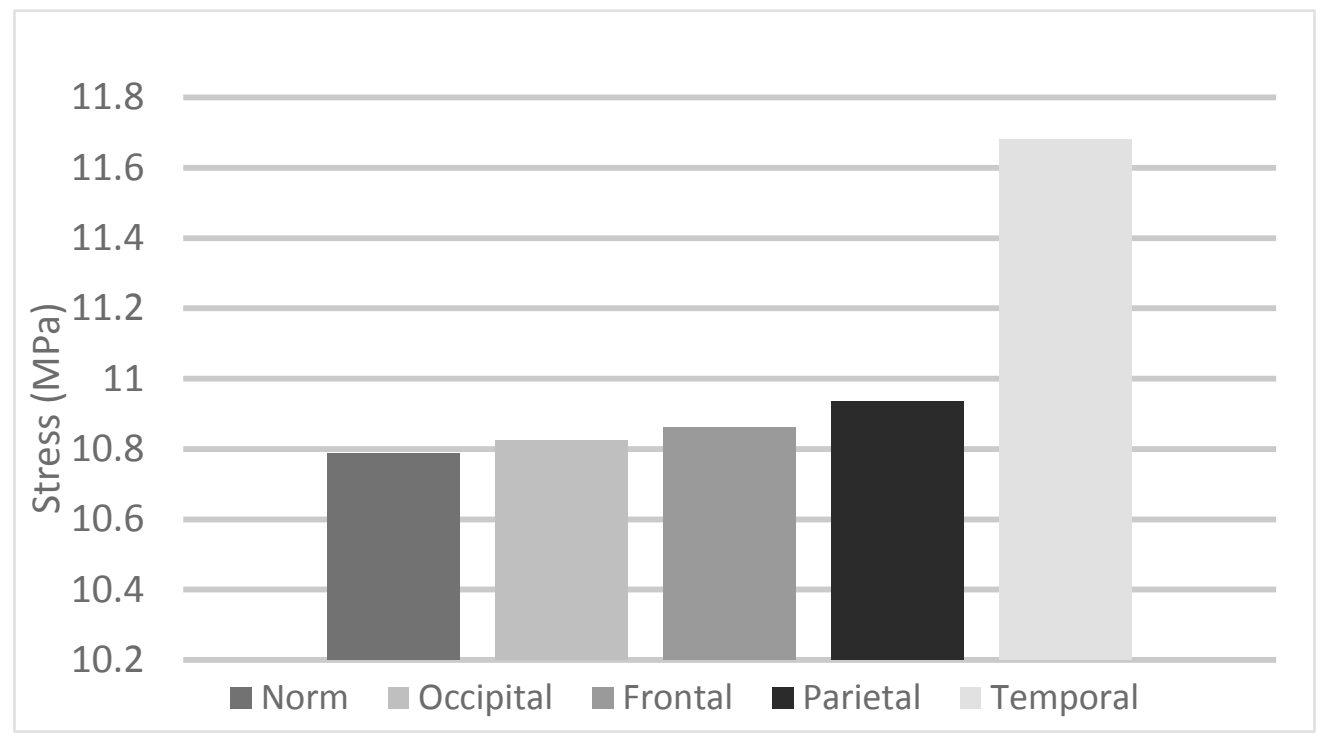

Figure 10. Global Mean von Mises Stress of different testing sites. 